\title{
Oportunidades, sostenibilidad y riesgos de los negocios informales de la comuna 10 de Cali
}

\author{
Julio César Ramírez Reyes, ${ }_{1}^{1}$ Gerardo Alexánder Vergara Mesa² \\ Universidad de San Buenaventura, Cali-Colombia
}

Recibido: febrero 10 de 2014 - Revisado: mayo 13 de 2014 - Aceptado: julio 7 de 2014

Referencia para citar este artículo: Ramírez-Reyes, J. C., \& Vergara-Mesa, G. A. (2014). Oportunidades, sostenibilidad y riesgos de los negocios informales de la comuna 10 de Cali. Revista Cientifica Guillermo de Ockham, 12(1), 35-41

\section{Resumen}

Esta investigación se desarrolló con el propósito de analizar los negocios informales que se llevan a cabo en la comuna 10 de la ciudad de Cali y cumplen con la condición de tener baja inversión inicial, ser autosostenibles en un futuro y estar en capacidad de trascender responsablemente hacia la formalidad. La metodología se fundamentó en juicio de expertos y en el diseńo y aplicación de una encuesta, teniendo en cuenta el último censo del Sector Industrial, Comercial y de Servicios realizado por el Dane y Cámara de Comercio de Cali en el año 2005. Se encontró que el $40 \%$ de los microempresarios encuestados realizan actividades en la División 15 Elaboración de Productos Alimenticios y Bebidas como elaboración de productos de panadería, lácteos, molinería. Entre tanto, el 17 \% en la División 19 Curtido y Preparado de Cueros, como fabricación de calzado y el 10 \% en la División 20 Transformación de la Madera, como fabricación de muebles para el hogar. Se concluye que el instrumento diseñado permitió identificar cuáles de los microempresarios cumplen con la condición de bajo costo y autosostenibilidad para trascender a la formalidad.

Palabras clave: Inversión, desempleo, economía, negocio, informal, emprendedores.

\section{Opportunities, sustainability and risks of informal business commune 10 Cali}

\section{Abstract}

The research was conducted in order to analyze business casual Comuna 10 Cali that meet the condition of having low initial investment and be self-sustaining in the future may also responsibly beyond formality. The methodology was based on expert judgment and the design and implementation of a survey, taking into account the last census in Industrial, Commercial Sector and Services performed by the Dane and the Chamber of Commerce of Cali in 2005. Was found that $40 \%$ of employers surveyed micro active in Division 15 Manufacture of Food Products and Beverages as preparation of bakery products, dairy products, grain mill. Meanwhile, 17\% in 19 Tanning and Leather Prepared as shoemaking division and $10 \%$ in Division 20 Wood Processing and manufacture of furniture for the home. We conclude that the instrument designed to identify which allowed the micro entrepreneurs meet the condition of lowcost, self-sustainability to transcend formality.

Keywords: Investment, unemployment, economy, business, casual, entrepreneurs.

* Este artículo fue elaborado en el marco del proyecto de investigación Análisis cualitativo y cuantitativo de los negocios de bajo costo y autosostenibilidad con fines de formalización con una inversión máxima de diez salarios minimos legales vigentes en el año 2013 en la ciudad de Santiago de Cali.

1. Contador Público Universidad de San Buenaventura. Magíster en Administración de Empresas Universidad del Valle. Docente de Finanzas Facultad de Ciencias Económicas Universidad de San Buenaventura Cali. Favor dirigir correspondencia a la carrera 24C \# 6-55 Oeste Edificio Gloria Apartamento 303 E-mail: jcramire@usbcali.edu.co.

2. Contador Público Universidad Libre. Magíster en Administración de Empresas Universidad del Valle. Docente de Costos Facultad de Ciencias Económicas Universidad de San Buenaventura Cali. E-mail: gerarve@usbcali.edu.co 


\section{La informalidad como fuente de ingreso}

El sector informal se define como el conjunto de actividades no sometidas a la regulación de las instituciones de la sociedad y dedicadas a la generación de ingresos, todo ello enmarcado en un ambiente social y legal en el que actividades similares sí están sometidas a regulación (International Labor Organization, 1972; Castells, Portes y Benton, 1989). Se define, entonces, como informales, todas aquellas actividades productivas de bienes y servicios lícitos no registradas ante las autoridades (Shneider y Enste 2000). La informalidad es un conjunto de unidades dedicadas a la producción de bienes y prestación de servicios que funcionan típicamente en pequeña escala y de acuerdo con una organización rudimentaria (Ochoa y Ordońez, 2004).

En la informalidad, las relaciones de empleo se basan en el parentesco o las relaciones personales y sociales y no en acuerdos contractuales que supongan garantías formales (Ochoa y Ordóńez, 2004). Los vendedores ambulantes, los trabajadores a domicilio y los vendedores en la vía pública, entre otros, son identificados habitualmente como miembros de este sector. La mayoría de ellos son trabajadores que no tienen contrato y no cuentan con seguridad social ni prestaciones sociales de ley.

Dos perspectivas enfocan actualmente la informalidad laboral: la estructuralista y la institucionalista (Uribe y Ortiz, 2006). La primera, hace énfasis en la limitación del sector productivo para generar empleo de buena calidad; la segunda, apunta a la opción que tienen los trabajadores y las empresas de cumplir o no las regulaciones institucionales. La informalidad es la única elección laboral para los individuos que no encuentran otras posibilidades a causa del desempleo o de otras restricciones del mercado laboral (Perry et al. 2007). En ciertos casos, la informalidad se convierte en un refugio involuntario (Mejía y Posada, 2007). La definición más completa de informalidad empresarial es la de no contar con un registro mercantil (Cárdenas y Mejía 2007).

Se han identificado dos causas por las que se puede dar la informalidad laboral. La primera se asocia directamente con el aumento del lucro y la segunda se relaciona con las condiciones de pobreza (Cordero, 2011). Los negocios informales se caracterizan por el valor mínimo de inversión inicial y el bajo costo de sus operaciones.

\section{Contexto de la informalidad empresarial en la ciudad de Cali}

La Cámara de Comercio de Cali, de la mano con el Departamento Administrativo Nacional de Estadística (dane) y las alcaldías de Cali y Yumbo, llevó a cabo en el año $2005^{3}$ un censo a fin de analizar el panorama del sector industrial, comercial y de servicios que presentaban estos municipios.

El censo permitió identificar las unidades económicas con registro mercantil vigente, aquellas que pagan impuesto de industria y comercio y las que cuentan con el registro único tributario (RUT). En la Tabla 1 se muestran los niveles de informalidad de las unidades económicas de la ciudad de Cali a partir de estos tres tipos de registros.

Tabla 1

Informalidad empresarial en la ciudad de Cali

\begin{tabular}{lcc}
\hline & Formal & Informal \\
\hline Registro mercantil & $56,8 \%$ & $43,2 \%$ \\
Industria y comercio & $47,6 \%$ & $52,4 \%$ \\
RUT & $56,5 \%$ & $43,5 \%$ \\
\hline
\end{tabular}

Fuente: Elaboración propia a partir del Censo Económico realizado en Cali y Yumbo (DANE, 2005).

En la ciudad de Cali fueron registradas 22.192 unidades economicas informales que equivalen al $43 \%$ del tejido empresarial. A nivel sectorial, el comercio representa el $46 \%$, la industria el segundo mayor grado de informalidad con el $40 \%$ y finalmente, el sector servicios representa el $38 \%$.

En cuanto al empleo, las unidades informales tienen el $21 \%$ del personal ocupado de la ciudad y de ellas solo el 4,1\% hacen aportes al sistema de seguridad (DANE, 2005), situación que implica un factor negativo en la calidad del empleo generado por las empresas informales. El $46 \%$ del personal ocupado por las unidades económicas informales son mujeres, participación que es menor en las unidades que sí cuentan con registro mercantil. En cuanto al tiempo de funcionamiento de las unidades económicas informales, se observa que cerca del $60 \%$ tienen de uno a dos años de operación y el $24 \%$ cuentan entre tres a cinco años (DANE, 2005). Lo anterior refleja los bajos niveles de subsistencia de las unidades informales.

Según el último censo económico de Cali-Yumbo (DANE, 2005) y el estudio de población para Cali y su área

3. En la Cámara de Comercio de Cali no hay registro de información más actualizada 
de influencia hecho por el Departamento Administrativo de planeación municipal de la ciudad de Cali (DAPM) y las Empresas Municipales de Cali (Emcali) la tasa de informalidad de la comuna 10 se encuentra entre el $41 \%$ y el $48 \%$.

\section{Método}

\section{Participantes}

La población objetivo está conformada por negocios informales o no convencionales de la comuna 10 , del sector industrial de la ciudad de Cali. Es importante mencionar que los criterios de selección de la comuna 10 como objeto de estudio para la presente investigación, se soportan básicamente en el hecho de ser una de las comunas más tradicionales de la ciudad de Cali, la cual según el censo económico del 2005 cuenta con uno de los porcentajes más altos en cuanto a informalidad, representado entre un $41 \%$ y un $48 \%$ del total.

Los 302 informales objeto de estudio fueron contactados mediante el método de observación directa. Los estudiantes del equipo de investigación, integrantes activos del programa de contaduría y del semillero de investigación en gestión de costos, para efectos del trabajo de campo visitaron la comuna 10 a fin de estructurar la información relativa a los participantes sobre la base de características como ser empresarios informales que se ubican estratégicamente cerca de los formales que desarrollan una misma actividad (en este caso industrial) y la tendencia a ubicarse en sectores considerados comerciales en los diferentes barrios objeto de estudio. Por último, se interrogó directamente a los empresarios formales que por lo regular conocen la competencia no formal.

La comuna 10 de Santiago de Cali cubre el 3,6 \% del área total de la ciudad y está conformada por dieciocho barrios: El Dorado, El Guabal, La Libertad, Santa Elena, Las Acacias, Santo Domingo, Jorge Zawadsky, Olímpico, Cristóbal Colón, La Selva, Departamental, Pasoancho, Panamericano, Colseguros Andes, San Cristóbal, Las Granjas, San Judas Tadeo I y San Judas Tadeo II. Estos barrios representan el 7,2\% del total de los barrios de la ciudad cuya población total asciende a 103.087 habitantes, de los cuales el 46,1\% son hombres y el 53,9\% mujeres, todos ellos agrupados en 27.062 viviendas. El estrato más representativo es el 3 y los niveles de educación distintivos son primaria y secundaria, según el departamento administrativo de planeación municipal.
Para determinar el tamaño de la muestra, se utilizó el muestreo aleatorio simple aplicado para una población calculada de 139 empresas informales (estimadas de acuerdo con el número de empresas formales del sector industrial en la comuna 10 de Cali y el porcentaje de informales en relación con el total de unidades económicas 2005, según los datos de la Cámara de Comercio para el 2010 y los datos del censo económico 2005). Lo anterior, sumado a un nivel de confianza del $95 \%$ y a un error de muestreo de 0,05 permitió establecer un objetivo de 102 encuestas como muestra.

\section{Instrumento}

Se diseñó una encuesta basada en la metodología de muestreo aleatorio simple para ser aplicada a microempresarios informales ubicados en la comuna 10 de la ciudad de Cali. Para el diseño, se contó con el apoyo de estudiantes de la quinta cohorte, promoción 2011, de la Especialización en Gerencia Estratégica de Costos de la Universidad de San Buenaventura Cali.

El primer diseńo de la encuesta constaba de cincuenta y tres preguntas. La prueba inicial de campo fue desarrollada por estudiantes de pregrado del programa de Contaduría Pública y por estudiantes adscritos al semillero de investigación en gestión de costos de la misma Universidad. Finalmente, la encuesta fue ajustada a dieciocho preguntas.

Para recabar información orientada a conocer la oportunidad y la sostenibilidad en el mercado, se preguntó acerca de la inversión inicial, su origen y los recursos financieros para sostener el negocio mes tras mes, todo ello con el fin de identificar si se ha requerido apalancamiento y de qué tipo, si el negocio del microempresario cubre sus necesidades y si este logra hacerse a algún tipo de ahorro. También se consultó información acerca de las actividades desarrolladas con mayor frecuencia en la informalidad en la comuna 10.

\section{Procedimiento de aplicación}

Para la aplicación de la encuesta se seleccionó un negocio informal por cada negocio formal radicado en el sector industrial e identificado en la base de datos de la Cámara de Comercio. La aplicación de la encuesta se hizo con la intervención de los estudiantes del programa de Contaduría Pública y del semillero de gestión de costos de la Universidad de San Buenaventura Cali, a quienes se les garantizó su seguridad y facilidad para los desplazamientos. Respecto a las consideraciones éticas, la encuesta se acompañó de un documento de consentimiento y 
confidencialidad de la información avalado por el comité de ética de la Universidad de San Buenaventura Cali.

\section{Preparación de datos}

Para el análisis de los negocios que cumplen con la condición de bajo costo y autosostenibilidad y que no superen la inversión inicial de diez salarios mínimos en Colombia al año $2012(566.700 \times 10=\$ 5.667 .000)$, se tiene que de 412 , solo 302 cumplen con las condiciones requeridas.

\section{Resultados}

De los 302 negocios informales, liderados en un $54 \%$ por hombres y en $46 \%$ por mujeres, el $42 \%$ ha sido ejecutado por personas mayores de cuarenta y cinco años y el $18 \%$ por personas cuyas edades están entre los cuarenta y cuarenta y cinco ańos, lo cual refleja que el $60 \%$ de los negocios informales han sido desarrollados personas de cuarenta años o más. Esto puede ser un indicador de falta de oportunidades para personas de este rango de edad y que habitan en la comuna 10, situación que muy probablemente sea similar en otras comunas de la ciudad de Cali. La mayor parte de las personas entrevistadas perdieron sus empleos o han pasado gran parte de su tiempo intentado ubicarse en un trabajo decente.

El instrumento permitió evidenciar que en la comuna 10 los microempresarios informales de las industrias manufactureras responden a las características estratégicas de producción definidas por la clasificación internacional industrial uniforme (CIIU). La taxonomía ha servido para clasificar los datos según el tipo de actividad económica que agrupa tipo de producción, empleo, ingreso y participación de la actividad económica de un país:

- Empresas dedicadas a la elaboración de productos alimenticios y bebidas de consumo masivo (productos de panadería, lácteos, molinería): $40 \%$.

- Empresas dedicadas a la fabricación de calzado: $17 \%$.

- Empresas dedicadas a la confección de prendas de vestir: $13 \%$.

- Empresas dedicadas a la transformación de la madera (en especial para la fabricación de muebles): $10 \%$.

En cuanto a la oportunidad de los habitantes de la comuna 10 de emprender negocios informales con poca inversión inicial, los resultados indican que el $52 \%$ de los empresarios iniciaron su negocio con una inversión entre uno y dos millones de pesos, el $16 \%$ lo hicieron con una inversión entre dos y tres millones y $14 \%$ con una inversión menor a un millón de pesos.

Se encontró que el dinero inicial o capital de plante para comenzar su negocio proviene de las siguientes fuentes:

1. Recursos propios y ahorros (69\%).

2. Aporte de un familiar (7 \%).

3. Préstamo personal (13\%).

4. Préstamo de una entidad financiera $(8 \%)$.

Lo anterior revela que los informales no son objeto prioritario para las entidades financieras por el riesgo que conllevan y las bajas esperanzas de éxito. Más de la mitad de los que notificaron que no les alcanzaba el dinero, reveló que solicitaba dinero prestado a familiares y amigos para aliviar sus dificultades financieras, mientras que un $28 \%$ del total de encuestados manifestó deber dinero a una persona natural y no a una entidad bancaria, lo que dejar ver la baja presencia del sector financiero en el apalancamiento de este renglón de la economía.

Una prueba de independencia entre el nivel de estudio y los recursos para mantener el negocio mes a mes, revela que el nivel de escolaridad no es una garantía para que los microempresarios accedan a los préstamos del sistema financiero. Con un valor de chi-cuadrado de Pearson de 14,99 para veinte grados de libertad y un valor de $p$ $=0,777$, no hay evidencia para concluir que el nivel de escolaridad y los recursos para mantener el negocio estén relacionados.

Para analizar la autosostenibilidad de los empresarios se utiliza el criterio del endeudamiento, bajo el cual un negocio es sostenible en su operación si sus ingresos permiten asumir los costos y gastos sin utilizar el crédito. En caso contrario, el negocio estaría sujeto a un préstamo o crédito. Según los resultados de la investigación, casi todos los encuestados se ven en la necesidad de solictiar crédito para solventar su operación. En la Tabla 2 se observa que los microempresarios informales dependen de un crédito para su actividad, por lo que están condenados al círculo vicioso del endeudamiento.

En la misma Tabla se evidencia una tendencia en las actividades en el sentido de que todas se apalancan principalmente en créditos personales. La actividad que más utiliza el crédito con entidades financieras es el de calzado de cuero. El uso del crédito gota a gota está particular- 
Tabla 2

Recursos financieros para mantener el negocio mes a mes

\begin{tabular}{|c|c|c|c|c|c|c|}
\hline Actividad & $\begin{array}{l}\text { Préstamos } \\
\text { personales }\end{array}$ & $\begin{array}{c}\text { Crédito con las } \\
\text { personas que le } \\
\text { suministran materia } \\
\text { prima }\end{array}$ & $\begin{array}{l}\text { Préstamos con entidades } \\
\text { cooperativas o financieras }\end{array}$ & $\begin{array}{l}\text { Préstamos } \\
\text { familiares }\end{array}$ & Otros & Total \\
\hline $\begin{array}{l}\text { Elaboración de productos } \\
\text { de panadería }\end{array}$ & 32 & 12 & 8 & 20 & 51 & 123 \\
\hline Prendas de vestir & 15 & 6 & 7 & 5 & 7 & 40 \\
\hline $\begin{array}{l}\text { Calzado de cuero, } \\
\text { marroquinería }\end{array}$ & 13 & 3 & 12 & 9 & 15 & 52 \\
\hline $\begin{array}{l}\text { Fabricación de muebles } \\
\text { para el hogar }\end{array}$ & 7 & 6 & 9 & 2 & 6 & 30 \\
\hline
\end{tabular}

mente generalizado en los microempresarios informales (otros recursos financieros), ya que se puede utilizar sin cumplir con los requisitos exigidos por el sector financiero.

Con un valor de chi-cuadrado de Pearson de 106,51 para setenta y ocho grados de libertad y un valor de $\mathrm{p}=$ 0,18 , se demuestra que la forma de obtener los recursos está relacionada con el tipo de negocio.

\section{Discusión}

Los microempresarios informales seleccionan actividades tradicionales que no requieren gran experticia para desarrollarse, pero sí mucho tiempo y disciplina. Si bien la elaboración de productos alimenticios es una arraigada tradición cultural vallecaucana, los resultados manifestaron que quienes desarrollan la actividad más común como es la elaboración y comercialización de pan no son originarios de la ciudad sino emigrantes de las regiones de Antioquia y Caldas, cuya concepción del trabajo se distingue por el tiempo de dedicación, el compromiso de salir adelante, la firmeza para sostenerse en la adversidad y los fuertes lazos de solidaridad intrafamiliar.

Los negocios informales no son objeto de crédito por parte del sector financiero formal y se sustentan con empréstitos no oficiales que conceden prestamistas ilegales identificados como los "gota a gota", lo que hace que la actividad caiga en un remolino crediticio sin fin que la hunde cada vez más en la informalidad y disminuye sus posibilidades de trascender hacia la formalidad. El sector financiero no considera dentro de sus objetivos estratégicos este tipo de negocios debido al riesgo que puede representar la recuperación de la cartera. Sin embargo, apoyar el desarrollo de ideas sostenibles y comprobadas puede significar una oportunidad para el sector financiero, el cual viene analizando de forma lenta pero sostenida la manera de respaldar este tipo de negocios sin incurrir en un riesgo alto.

La carencia de estrategias de apoyo por parte del sector financiero a informales con altas probabilidades de desarrollo y la ausencia del Estado son obstáculos poderosos para el desarrollo colectivo de este tipo de negocios. El sector gubernamental requiere generar políticas públicas de mayor alcance que les permitan a este tipo de microempresarios tener vigencia en el mercado, trascender hacia la formalidad y crecer en ella.

No obstante, para el logro de este objetivo los beneficios iniciales no deben estar centrados solo en los descuentos de renovación de la matrícula mercantil, como fue lo aplicado por la Ley 1429 de diciembre 29 de 2010, cuya vigencia cubrió la investigación actual. Sin dejar de ser un estímulo importante para la formalización, esta ley no es lo suficientemente robusta para mantener vigente a los microempresarios durante un tempo adecuadamente largo para su consolidación debido a los altos impuestos y costos que deben asumir en la formalidad. Ello es razón suficiente para que las políticas públicas que se generen, amén de ser estratégicas y responsables para el fisco nacional, sean lo suficientemente atractivas para los microempresarios al punto de que haga germinar en ellos el convencimiento de que es mejor estar en la formalidad que en la informalidad y logren en el mediano o largo plazo, el impulso de microempresas que sin duda contribuirán a la disminución del nivel de pobreza, el desempleo y la inseguridad y produzcan, adicionalmente, mayores ingresos a las arcas de la nación.

Lo inequitativo del sistema hace que los informales no sean tenidos en cuenta para el desarrollo regional. Una de las estrategias vigentes que busca el desarrollo de la economía local son los clústeres, de los cuales se destaca en el Valle del Cauca el del azúcar. 
Consecuente con lo anterior, la Cámara de Comercio de Cali con base en un estudio oficial de la Gobernación del Valle identificó cinco clústeres que intenta promover desde diferentes frentes: bioenergía, proteína blanca, salud, belleza y bienestar y macrosnacks (este último relacionado directamente con los resultados de la investigación, cuya actividad principal es la elaboración de productos alimenticios y bebidas) los cuales podrían desarrollarse de manera conjunta en un futuro, ya que al iniciar con productores consolidados estos jalonarían dentro de un clúster tanto horizontal como vertical a los más pequeños.

Como reto de la región, los clústeres pretenden vincular los diferentes actores del Valle del Cauca y para ello demandan que los empresarios, los microempresarios y sobre todo las pyme, deben estar altamente capacitados para afrontar este tipo de desafíos. Es una oportunidad conjunta del Estado, el sector privado y la academia para unirse y ser participantes activos de este tipo de iniciativas que, sin duda, promueven un desarrollo conjunto, equitativo y responsable, al tiempo que disminuye el riesgo de que pequeños capitales se pierdan en emprendimientos poco viables y sin trascendencia.

Mediante proyectos de investigación a informales, la academia podría aprovechar los soportes para una formalidad responsable y sostenible y los programas de capacitación para aquellos negocios poco riesgosos y más viables que se articulen a los proyectos productivos que se han identificado para la región y aquellos factibles de desarrollar alternativamente, de acuerdo con los diagnósticos a las diferentes comunas de la ciudad.

El sector informal carece de información acerca de la viabilidad de ideas de negocio para plasmar en una comunidad. De acuerdo con los resultados de la investigación, uno de los problemas que surgen al seleccionar una idea de emprendimiento informal y su desarrollo hacia la formalidad, radica en buena parte en la falta de información sobre los asuntos o ideas de negocio que sean viables, constituyan una necesidad real de una comunidad y logren cierto grado de crecimiento y madurez.

En resumen, cabe afirmar que es imperativo llevar a cabo un trabajo conjunto y constante entre la universidad, las empresas y el Estado que beneficie no solo a las empresas que han logrado cierto grado de madurez formal, sino también a aquellas que a partir de la informalidad y en su tránsito a la formalidad, puedan llegar a formar parte fundamental del sostenimiento mayoritario de la economía local y nacional.

\section{Referencias}

Cárdenas, M. \& Mejía C. (2007). Informalidad en Colombia: nueva evidencia. Revista Working Papers Series, 35(1), 1-42.

Castells, M., Portes, A. \& Benton, L. (1989). The Informal Economy: Studies in Advanced and Less Developed Countries. Baltimore (MD): Johns Hopkins University Press.

Calleja, F. J. (2013). Costos. México: Editorial Pearson.

Cordero, M. (Julio del 2011). El fenómeno de la informalidad en Colombia. Recuperado de http://primernombre.com/ images/pdf/e0020.

Delgado, C. (2007). La microempresa de punta en Colombia. Bogotá: Editorial Universidad Externado de Colombia.

García, G. A. (2010). Evolución de la informalidad laboral en Colombia: determinantes macro y efectos locales. Archivos de economía.

Helfert, E. (2000). Análisis de las decisiones sobre las inversiones de capital. En: Técnicas de análisis financiero. Madrid

Horngren, C. (2012). Contabilidad de costos. México: Pearson Educación.

International Labor Organization (1972). Employment, Income and Equality: A Strategy for Increasing Productivity in Kenya.

Johnson, R. (2001). Métodos alternativos de financiamiento. Administración financiera. Madrid

Lowney, C. (2004). El liderazgo al estilo de los jesuitas. Bogotá. Grupo Editorial Norma.

Mejía, D., \& Posada, C. E. (2007). Informalidad: teoría e implicaciones de política. Bogotá: Editorial Banco de la República.

Perry, G., Maloney, W., Arias, O., Fajnzylber, P., Mason, A. \& Saavedra-Chanduvi, J. (2007). Informality: Exit and Exclusion. Washington D.C.

Santa María, M. \& Rozo, S. (2009). Análisis cualitativo y cuantitativo de la informalidad empresarial en Colombia. Revista Desarrollo y sociedad. p. 276.

Schneider, F. (2002). The Informal Economy in the EU-Accession and Instability Pact Countries: Size, Scope and Challenges to the process of EU Enlargement. Centro de Investigación de Europa del Este.

Schneider, F., \& Enste. D. H. (2000). Shadow economies: Size, causes, and consequences. Journal of Economic Literature, 38(1): 77-114.

Torres, C. A. (2009). Ciudad informal colombiana: barrios construidos por la gente. Santa Fe de Bogotá. Editorial Unal.

Uribe, J. I. \& Ortiz, C. H. (2006). Informalidad laboral en Colombia. Evolución, teoría y modelos. Cali: Editorial Universidad del Valle. 
Ochoa-Valencia, D. \& Ordóñez A. (2004). Informalidad en Colombia. Causas, efectos y caracteristicas de la economia del rebusque. Revista Estudios Gerenciales, 20(90), 42-45.

Varela, R. (2001). Innovación empresarial: arte y ciencia en la creación de empresas. Bogotá: Pearson Educación.
Wheelen, T. \& Hunger, D. (2007). Aspectos estratégicos en proyectos empresariales y pequeñas empresas. En: Administración estrategia y política de negocios. México. 